\title{
Nerocila acuminata (Isopoda: Cymothoidae) como parásito de cápsulas ovígeras de Rostroraja texana: lista de hospederos conocidos
}

\section{Nerocila acuminata (Isopoda: Cymothoidae) as an egg capsule parasite of Rostroraja texana: list of known hosts}

\author{
Luis Fernando Del Moral-Flores ${ }^{1 *}$, Luis Alfredo Sotelo-Sánchez ${ }^{1}$ y Manuel Ortíz ${ }^{2}$
}

\begin{abstract}
RESUMEN
Los isópodos de la familia Cymothoidae se caracterizan por ser ectoparásitos de una gran variedad de especies marinas, que incluyen al grupo de los tiburones y rayas. Sin embargo, las interacciones con este grupo son poco conocidas. Así, el presente trabajo tiene como objetivo evidenciar el primer registro de la asociación de cuatro isópodos hembras de N. acuminata (20$28 \mathrm{~mm}$ longitud total, $15-18 \mathrm{~mm}$ de ancho) con dos cápsulas ovígeras de $R$. texana encontradas en el estómago del tiburón martillo común $S$. lewini. La captura del tiburón se realizó el 17 de febrero de 2019 en la pesca artesanal de Salinas Punta Roca Partida, sur de Veracruz, México. Se presenta, además, una lista de 67 especies nominales de peces hospederos, así como algunos peces no identificados, incluidas en 36 familias, 19 órdenes y dos clases, registrados para $N$. acuminata, típica del Atlántico occidental, y su congénere del Pacífico oriental, $N$. californica. Esto demuestra que ambas especies de isópodos tienen varios peces hospedantes; sin embargo, se observa preferencia por las especies demersales, al destacar la familia Sciaenidae.
\end{abstract}

Palabras clave: Batoidei, ectoparásito, Elasmobranchii, golfo de México, isópodo

\begin{abstract}
Isopods of the Cymothoidae family are characterized for being ectoparasites of a wide variety of marine species including the shark and ray group. However, little is known of their interaction with this group. Consequently, this paper is aimed to present the first record of the association of four female $N$. acuminata isopods (20-28 mm total length, 15-18 mm wide) with two egg

1 Laboratorio de Zoología, Facultad de Estudios Superiores Iztacala, Universidad Nacional Autónoma de México (UNAM). Av. De los Barrios N. ${ }^{\circ}$, Los Reyes Iztacala, C. P. 54090 Tlalnepantla, Estado de México, México.

2 Laboratorio de Crustáceos, Facultad de Estudios Superiores Iztacala, Universidad Nacional Autónoma de México (UNAM). Av. De los Barrios N. ${ }^{\circ}$ 1, Los Reyes Iztacala, C. P. 54090 Tlalnepantla, Estado de México, México. delmoralfer@comunidad.unam.mx* ORCID: https://orcid.org/0000-0002-7804-2716, sotelos.alfredo@gmail.com ORCID: https://orcid.org/0000-0001-5798-5048, ortiztouzet@yahoo.com ORCID: https://orcid.org/0000-00026985-8019
\end{abstract}


capsules of $R$. texana, found in the stomach of a $S$. lewini common hammerhead shark. The shark was captured on February 17, 2019 using artisanal fishing in Salinas Punta Roca Partida, southern Veracruz, Mexico. A list of 67 nominal host fish species as well as some unidentified fish is presented including 36 families, 19 orders, and two classes, registered for N. acuminata, which is characteristic of the western Atlantic, and its eastern Pacific counterpart, $N$. californica. This demonstrates that both isopod species have several host fish; however, a preference for demersal species is observed, highlighting the Sciaenidae family.

Keywords: Batoidei, ectoparasite, Elasmobranchii, Gulf of Mexico, isopod

\section{INTRODUCCIÓN}

Los isópodos de la familia Cymothoidae son ectoparásitos de varias especies de peces marinas, estuarinas y dulceacuícolas (Smit et al. 2014). El género Nerocila Leach, 1818 cuenta con al menos 65 especies válidas (Trilles et al. 2013). En el Atlántico occidental, cuatro de ellas tienen distribución alopátrica. Nerocila lanceolata se extiende de nueva Inglaterra a Panamá, incluye a Cuba y las Bermudas; Nerocila fluviatilis de Brasil a Trinidad y Tobago; Nerocila benrosei del norte de las Bahamas y posiblemente Bermuda (Bunkley-Williams \& Wi1liams, 1999); y Nerocila acuminata con distribución más amplia y que en fase adulta se considera ectoparásito de peces marinos, incluye tiburones y rayas (Elasmobranchii), tanto pelágicos como demersales, los cuales se adhieren a la piel en la base de las aletas dorsal, pélvica y caudal, además de introducirse a los arcos branquiales y boca (Brusca, 1981; Segal, 1987).

Se han reportado 42 especies de elasmobranquios que son parasitadas por isópodos (Moreira \& Sadowsky, 1978; Benz \& Bullard, 2004; Caira et al. 2012). Estos pertenecen a las familias Aegidae, Cirolanidae, Corallanidae, Gnathiidae y Cymothoidae (Caira $\&$ Healy, 2004). Algunos de estos pueden parasitar a más de una especie de tiburón o raya. Así, en el Atlántico occidental Natatolana borealis se ha encontrado adherida a la piel, cavidad pericárdica y ventrículo o cono arterioso de al menos ocho especies de tiburones (Bird, 1978). Mientras que tres especies de isópodos (Aega sp., Aegaphales sp. y Cirolana sp.) se han reportado que infestan a dos especies de tiburones de aguas profundas (Shipley et al. 2017).

Los registros preexistentes de $N$. acuminata y $N$. californica que parasitan a peces son poco documentados, además, se han mezclado los registros que las consideran una sola especie (Brusca, 1981; Kensley \& Shotte, 1989; Carrillo-Colín et al. 2016). Sin embargo, no existen trabajos que reporten al isópodo $N$. acuminata que parasita las cápsulas ovígeras de Rostroraja texana (Chandler, 1921), por 
lo que el objetivo del presente estudio es informar por primera vez esta asociación, encontrada indirectamente en el estómago de un tiburón martillo común capturado en la región costera de Los Tuxtlas, Veracruz, golfo de México, así como presentar una lista actualizada de los peces hospederos de $N$. acuminata y $N$. californica.

\section{MATERIALES Y MÉTODOS}

El material analizado proviene de un tiburón martillo Sphyrna lewi$n i$ capturado con un palangre (línea madre de $1500 \mathrm{~m}$, orinques de $2 \mathrm{~m}$, canastas de $100 \mathrm{~m}$ de profundidad, reinales de 60-200 m y anzuelos del número 9/0; Fig. 1) el 17 de febrero de 2019 frente a la planicie costera en el municipio San Andrés Tuxtla, al sur de Veracruz, $18^{\circ} 42^{\prime} 18.3^{\prime}$ ' $\mathrm{N}-95^{\circ} 14^{\prime}$ 38.8' W, golfo de México, por pescadores ribereños de la comunidad de Salinas Roca Partida (Fig. 2).

El hospedero fue hallado como parte del espectro trófico del tiburón martillo. Se encontraron dos cápsulas ovígeras de $R$. texana las cuales tenían fuertemente adheridas cuatro especímenes hembras de N. acuminata. Dichos isópodos junto con las capsulas ovígeras se fijaron con formol al $10 \%$ y preservaron en alcohol al $70 \%$. Se trasladaron al Laboratorio de Zoología en la Facultad de Estudios Superiores Iztacala para identificarlos taxonómicamente por medio de claves especializadas (Brusca, 1981; Kensley \& Schotte, 1989; Trilles, 1991; McEachran \& Fechhelm, 1998). La validación del estatus taxonómico actual de los isópodos sigue a la propuesta de Boyko et al. (2020).

Finalmente, se elaboró una lista de hospederos conocidos para N. acuminata y $N$. californica, con los registros previos (Brusca, 1978, 1981; Brusca \& Iverson, 1985; Álvarez-León, 1981; Williams \& Bunkley-Williams, 2003; Carrillo-Colín et al. 2016). Se actualizó el estado taxonómico de las especies de ícticas con ayuda de Fricke et al. (2020) y se ordenaron a partir de la clasificación de van der Laan et al. (2019).

\section{RESULTADOS}

Los cuatro isópodos colectados pertenecen a la especie $N$. acuminata, que presenta la siguiente posición taxonómica.

\section{TAXONOMÍA}

Filo Arthropoda von Siebold, 1848

Subfilo Crustacea Brünnich, 1772

Clase Malacostraca Latreille, 1802

Subclase Eumalacostraca Grobben, 1892

Superorden Peracarida Calman, 1904

Orden Isopoda Latreille, 1817

Suborden Flabellifera Sars, 1882

Familia Cymothoidae Leach, 1814

Género Nerocila Leach, 1818

Nerocila acuminata Schioedte \&

Meinert, 1881

(Fig. 3) 

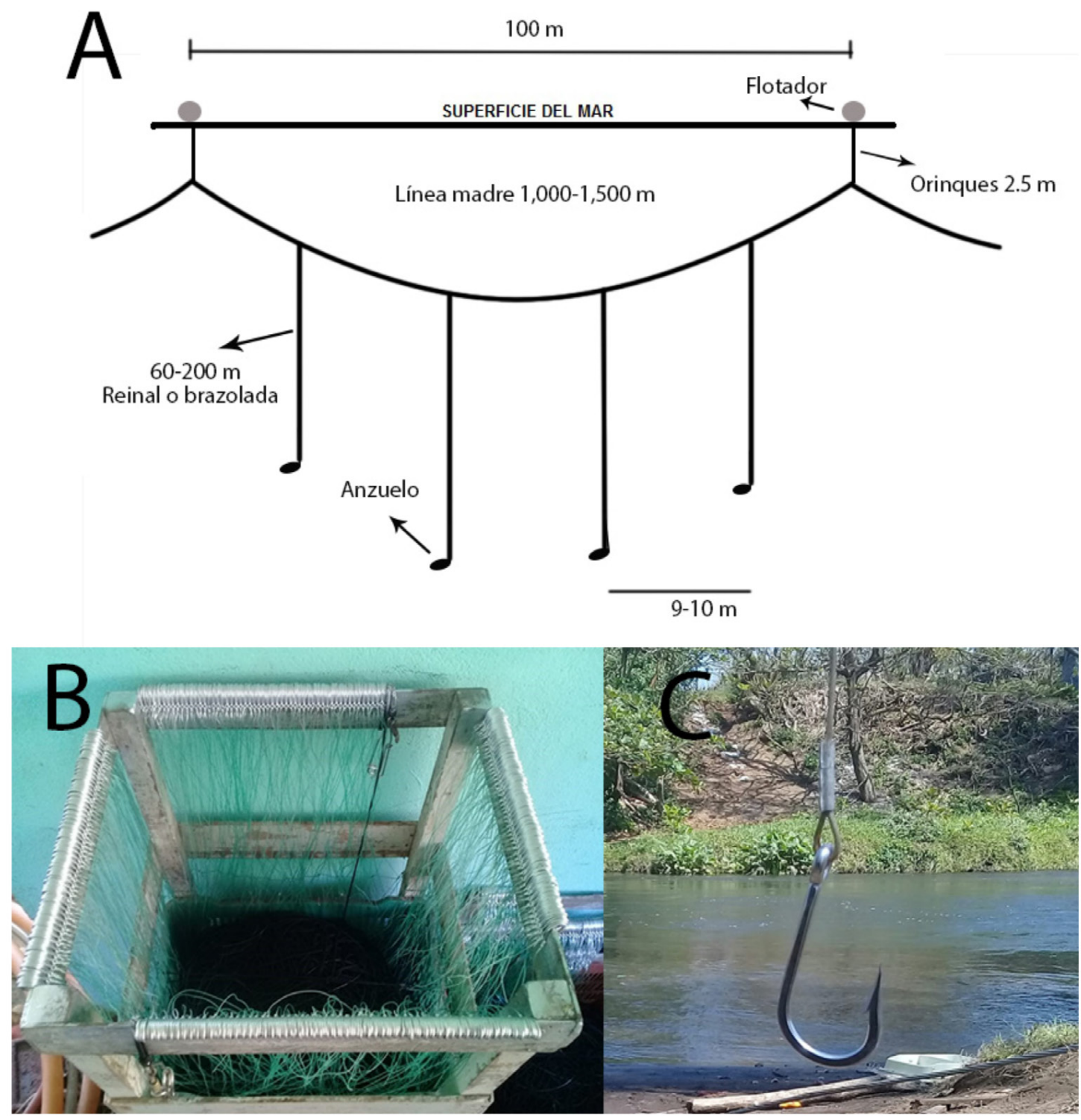

Fig. 1. A) Esquema y características del palangre utilizado por los pescadores ribereños de la comunidad de Salinas Roca Partida, B) arte de pesca bajo resguardado antes de su uso, y c) anzuelo del número $9 / 0$ utilizado en el palangre

Fig. 1. A) Design and characteristics of the longline used by the Salinas Roca Partida fishermen, B) Fishing gear protected before use, and C) Hook number 9/0 used in the longline 


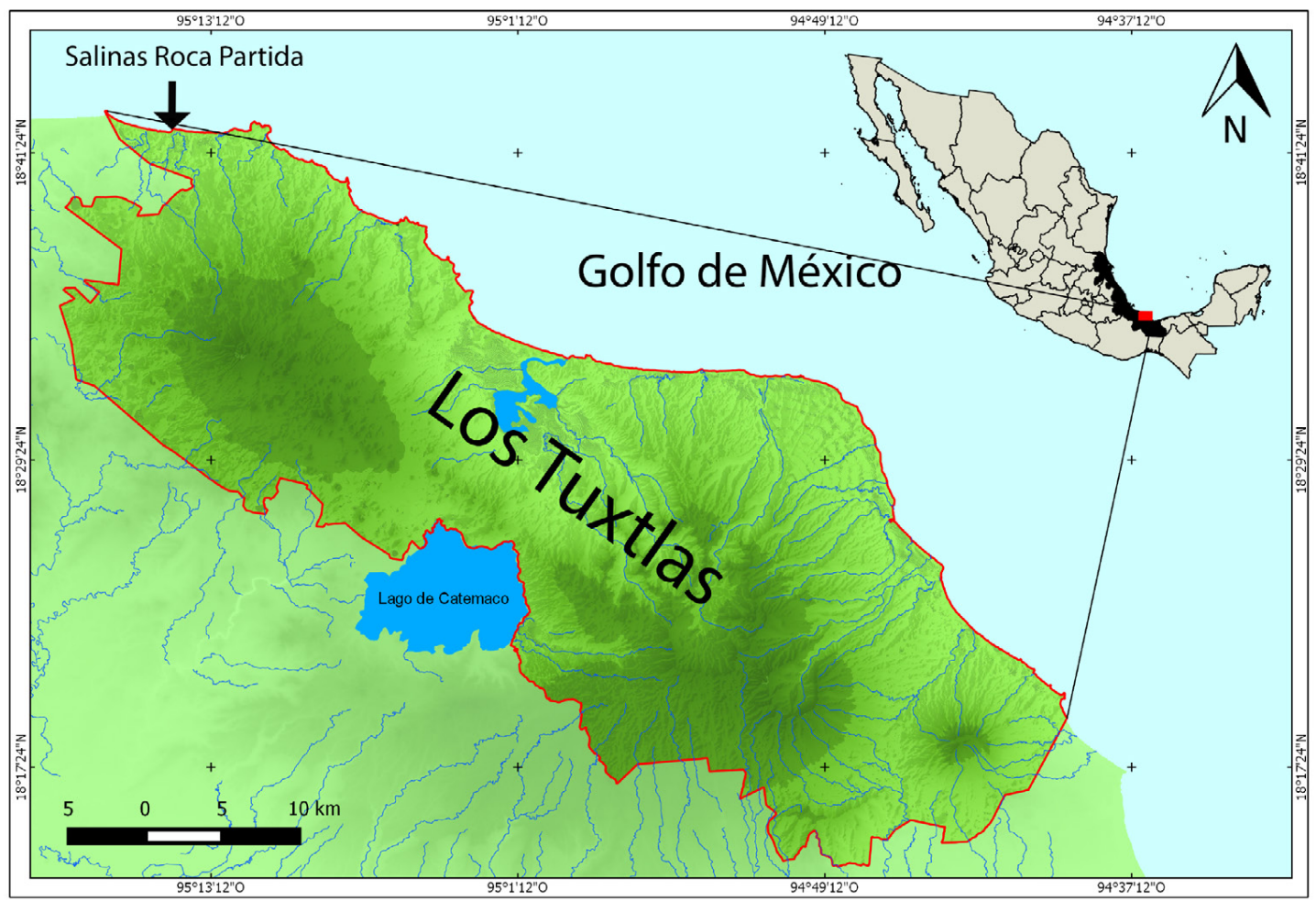

Fig. 2. Mapa de la región de los Tuxtlas, suroeste del golfo de México, la flecha indica el área de estudio de la comunidad pesquera de Salinas Punta Roca Partida, Veracruz, México

Fig. 2. Map of the Tuxtlas region, southwest of Gulf of México. The arrow indicates the study area in the fishing community of Salinas Punta Roca Partida, Veracruz, Mexico

Características del hospedero: Sphyrna lewini (Griffith \& Smith, 1834), macho maduro de $2.9 \mathrm{~m}$ de LT, capturado a una profundidad cercana a los $60 \mathrm{~m}$, fecha de captura 17 febrero 2019; dos capsulas ovígeras de $R$. texana (Chandler, 1921), $10.5 \mathrm{~cm}$ de altura, $6 \mathrm{~cm}$ de ancho.

Material examinado: cuatro hembras adultas, longitud total 20-28 mm, ancho 15-18 mm, localidad: Salinas Punta Roca Partida, San Andrés Tuxtla, México; colectado externamente de las cápsulas ovígeras de $R$. texana.

La compilación bibliográfica antes consultada, así como la actualización y validación de los registros se realizó por medio del proyecto de Fricke et al. (2020) permitió referir 67 

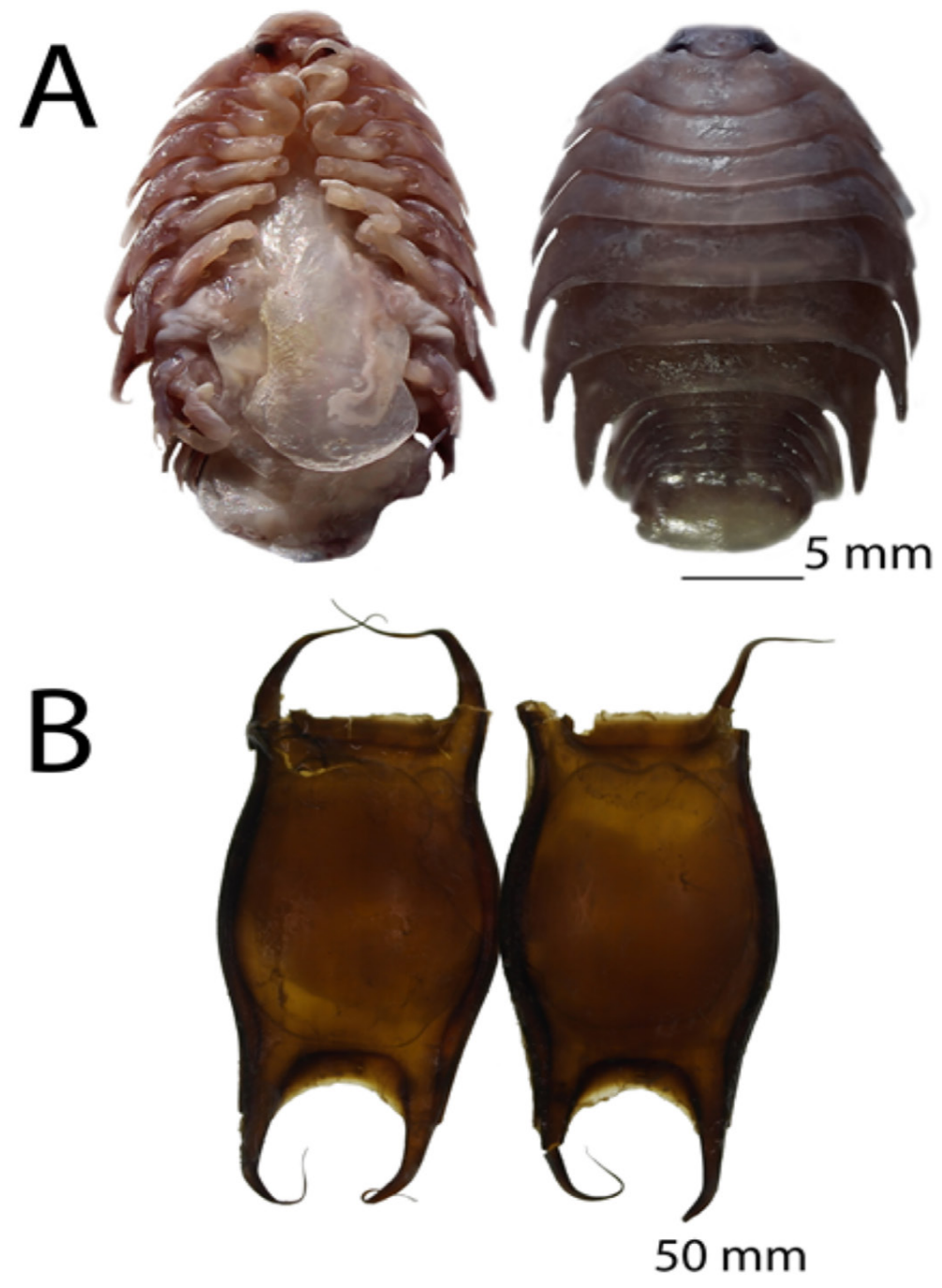

Fig. 3. Ejemplar hembra de Nerocila acuminata (A) asociados a las cápsulas ovígeras de Rotroraja texana (B)

Fig. 3. Female specimen of Nerocila acuminata (A), associated with Rostroraja texana ovigerous capsules (B)

especies válidas de peces hospederos, última especie de isópodo presentó 46 que incluye algunas no identificadas, especies de hospederos, se destacan pertenecientes a 32 familias, 18 órde- los asociados con la familia Sciaenines y dos clases (Cuadro 1), tanto para dae (9 especies) y Serranidae (7).

$N$. acuminata y $N$. californica. Esta 


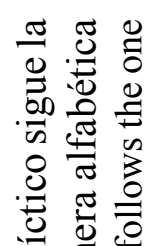

을 疋

कू ठै

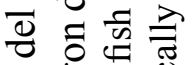

흐 흐용

헝

흥ㅎํ

क

$\frac{\pi}{0}$.

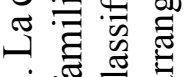

एं क्षै

跑悉

웡

¿ \& :

之

入 8 \&

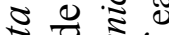

उ.

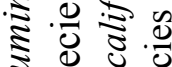

ड

을 चี

공

$\dot{\bar{\sigma}} \Xi E$

ช 히호

ช ฮ :

गั

月

ठ ฐ

क 14 要

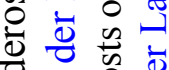

व 8 के

क्ते

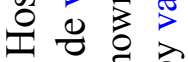

표

o क

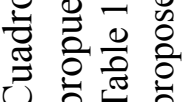

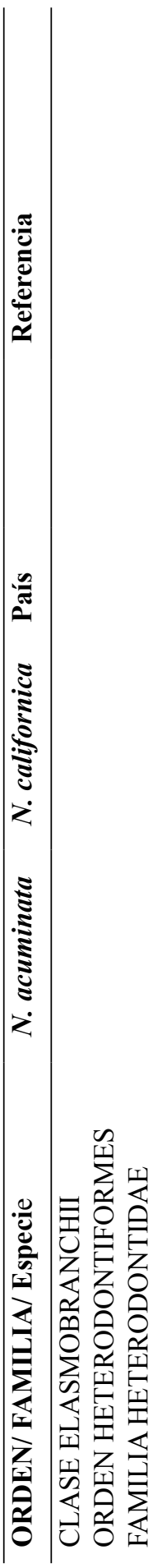

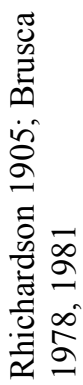

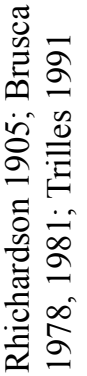

S

$x$

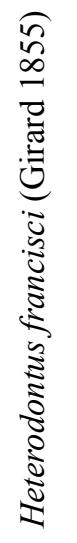

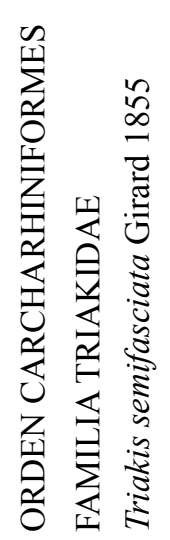

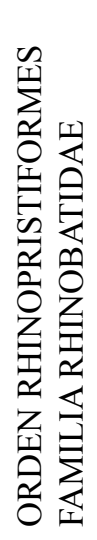

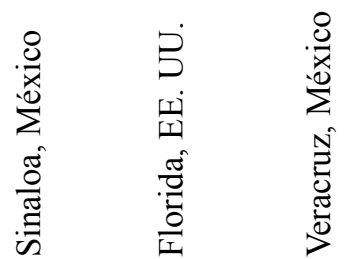
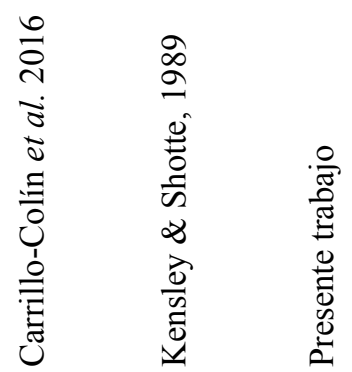

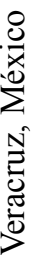
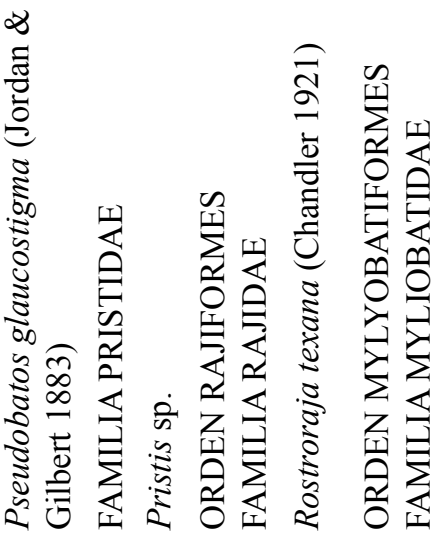

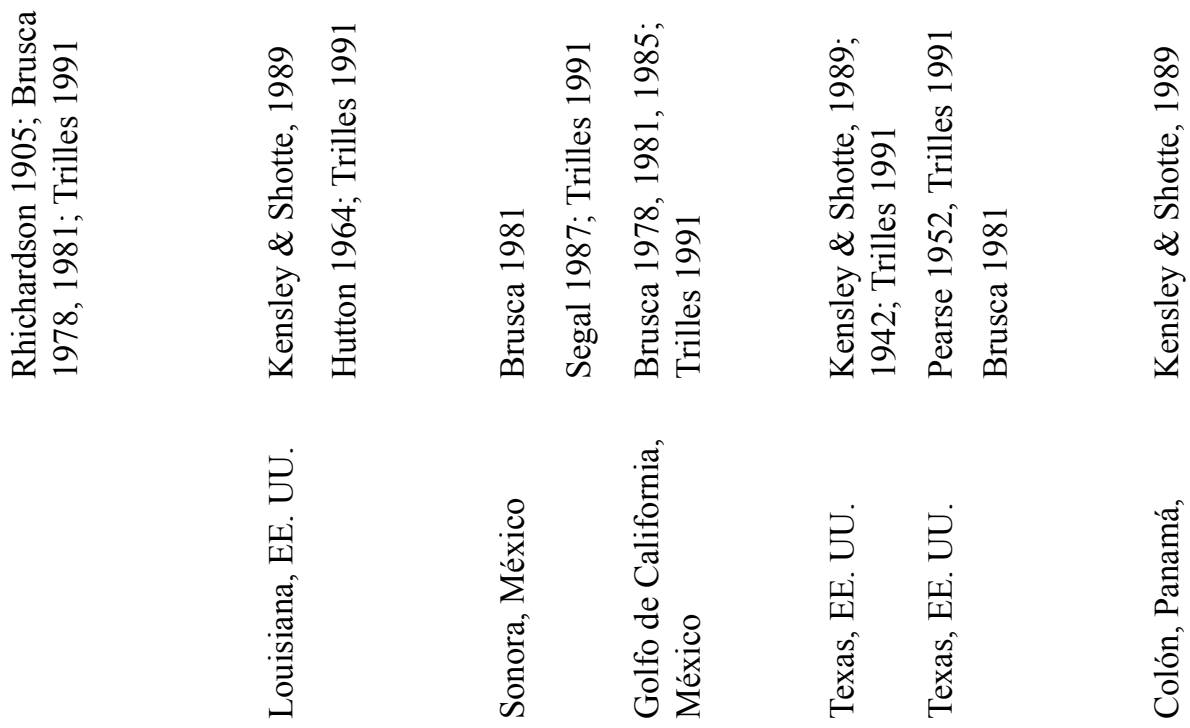

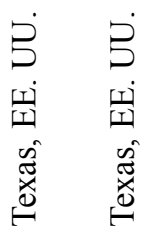

อี

$\Varangle$
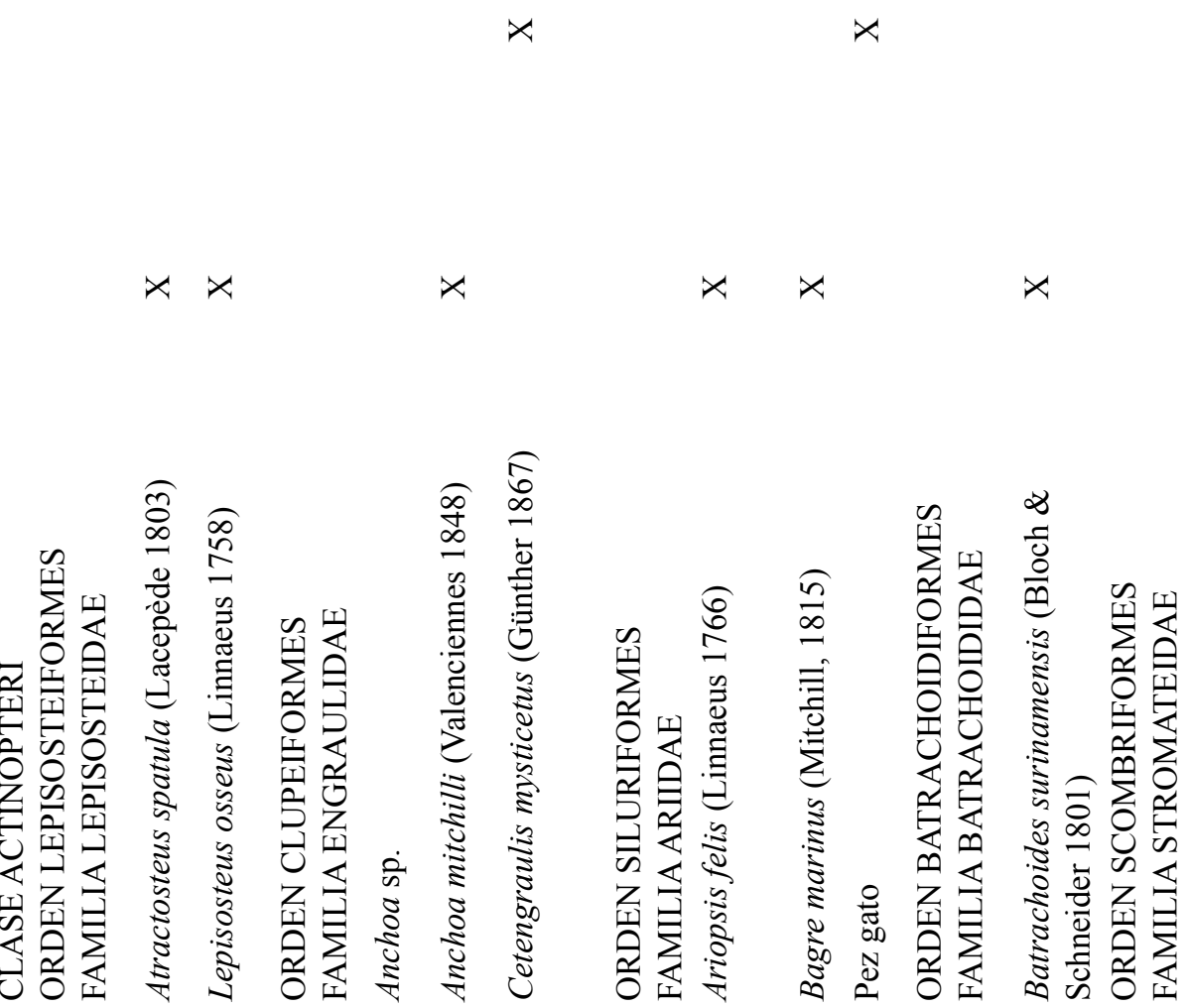


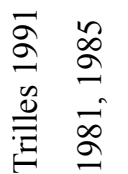

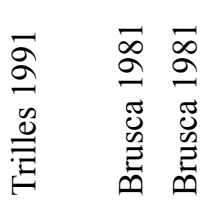

咅器

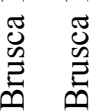<smiles>C#CC(=O)C#CC#CC(=O)C#C</smiles>

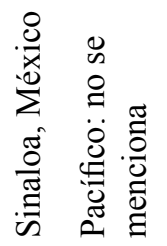

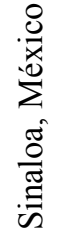

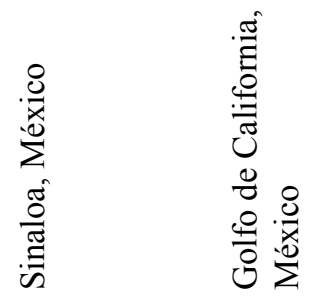

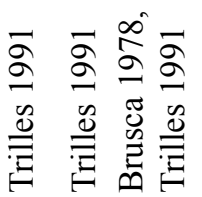

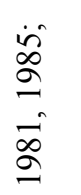

苞

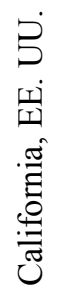

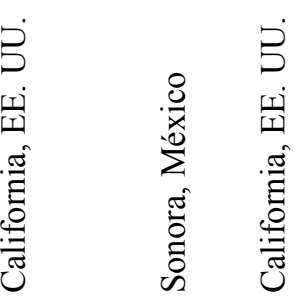

$x \times$<smiles>[Y]C1CC1</smiles><smiles>C1CCC1</smiles><smiles>[V]</smiles>

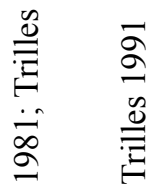

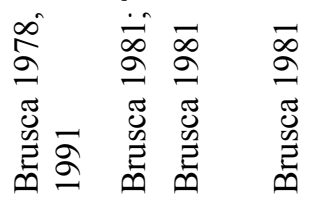
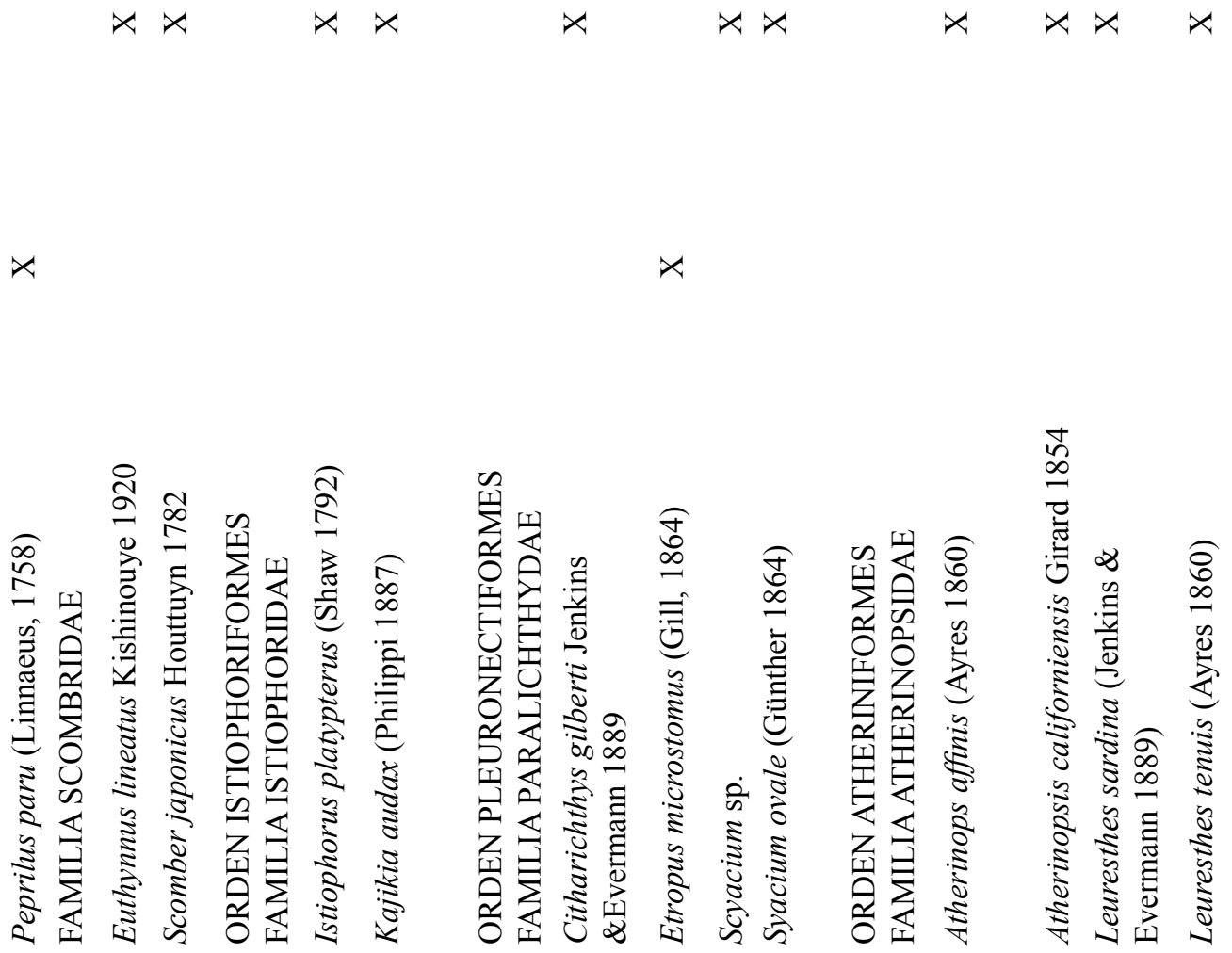

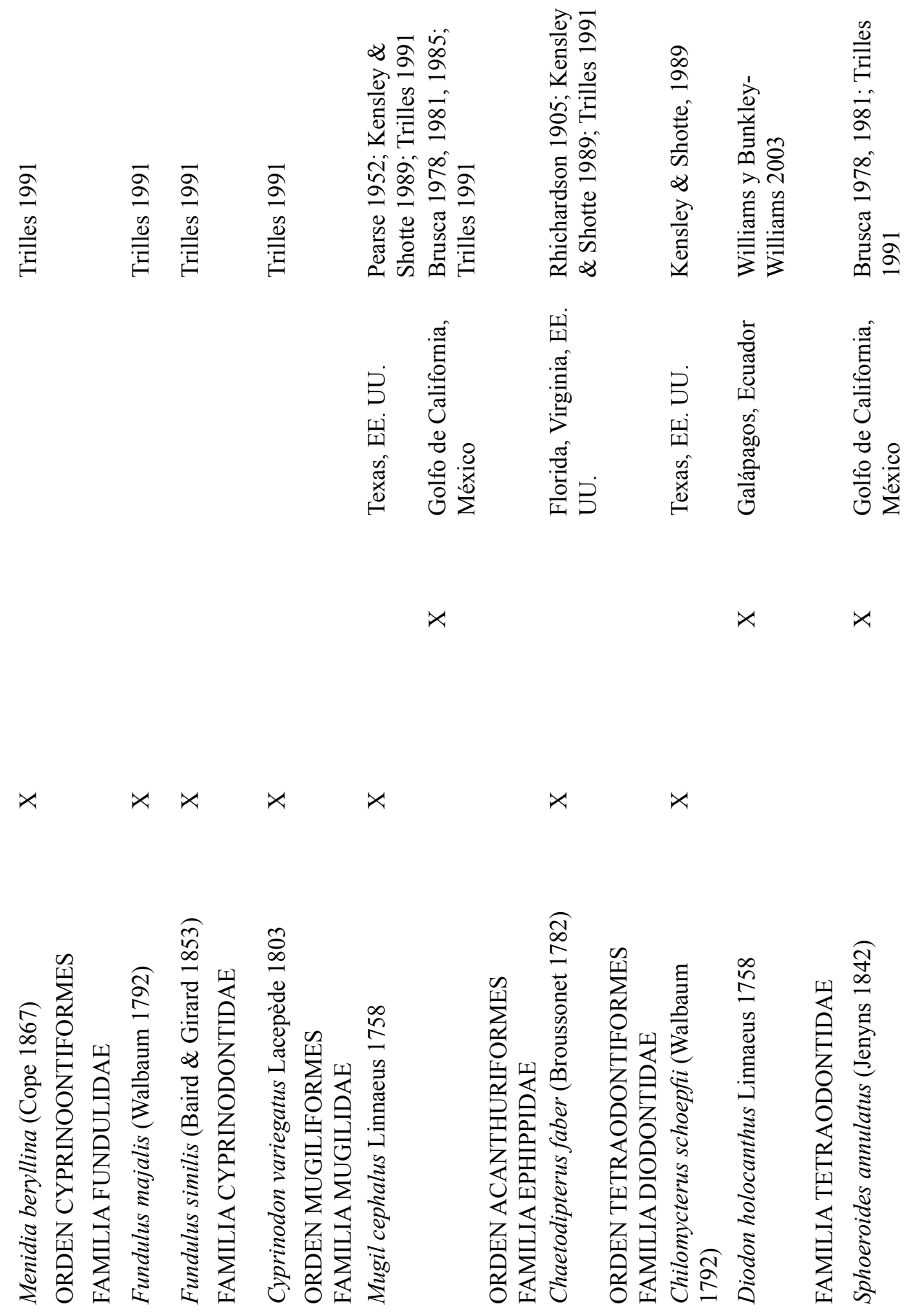

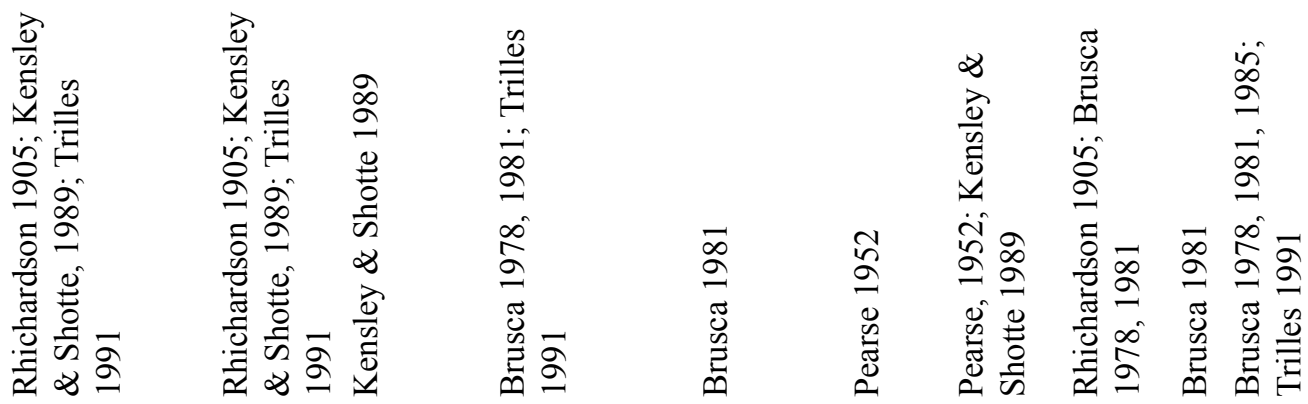

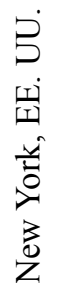
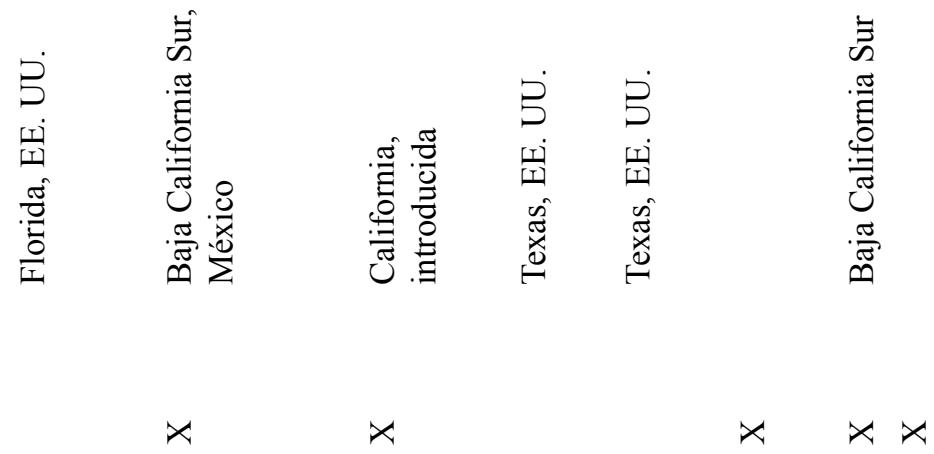

$x$
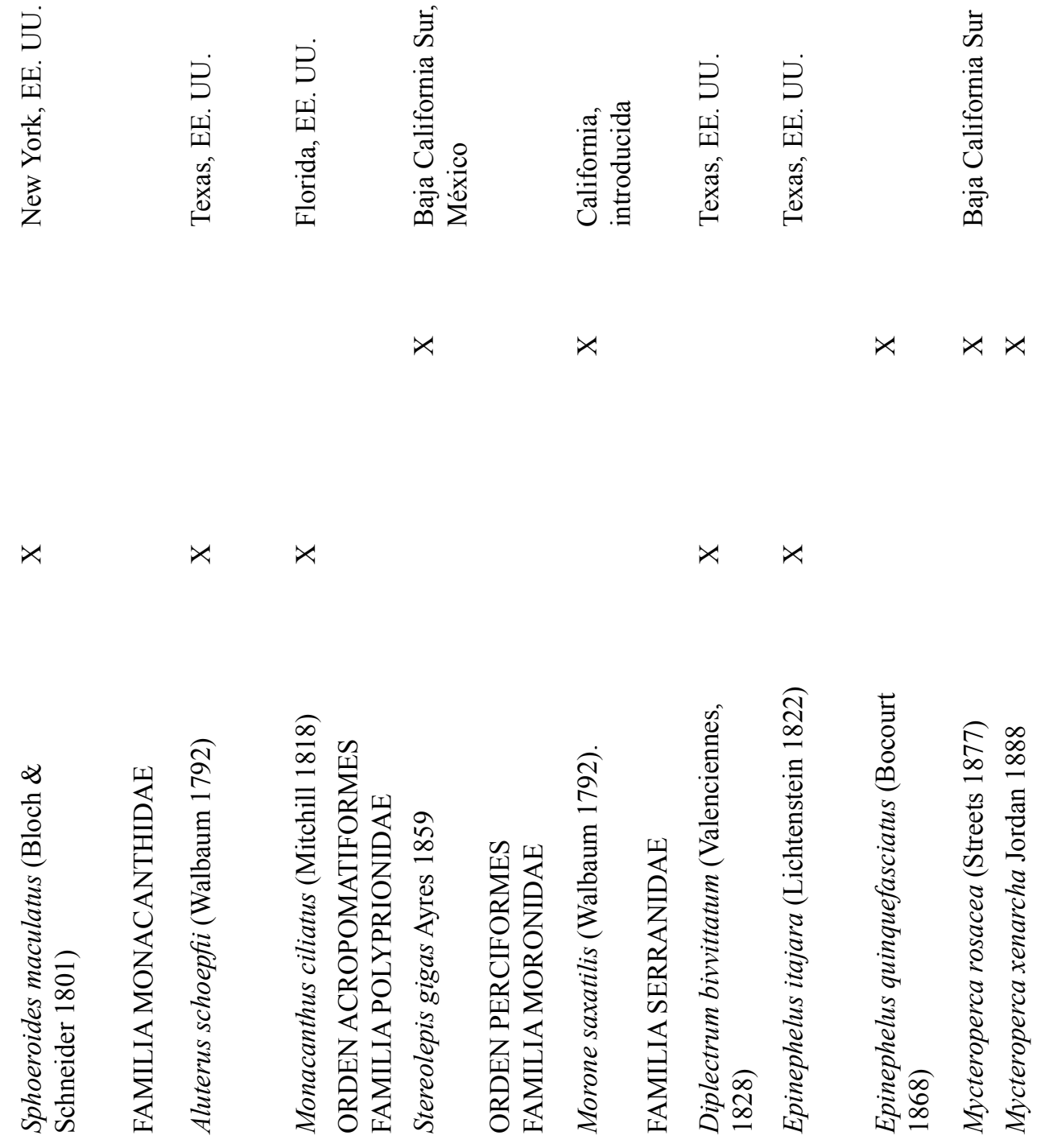
苞

in के

ชิ을

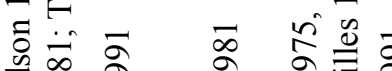

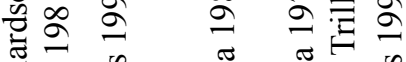

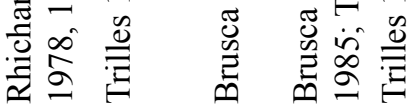

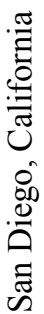

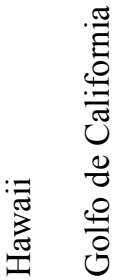

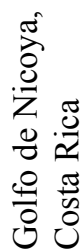

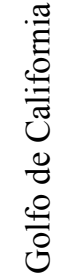

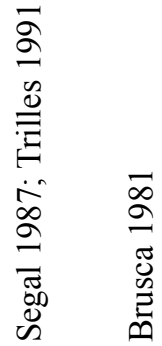

$\stackrel{\infty}{\approx}$

एँ

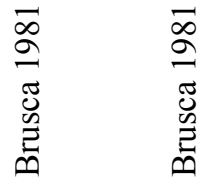

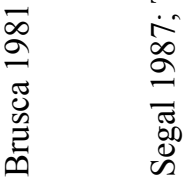

$\stackrel{\infty}{\infty} \dot{\infty} \quad \vec{\infty}$

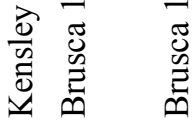

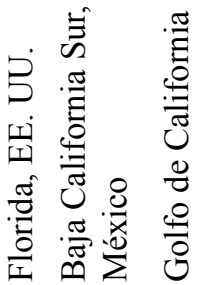

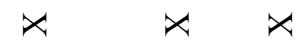

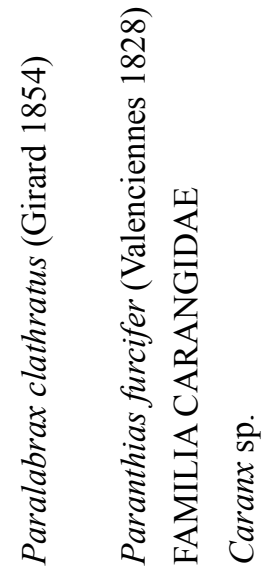

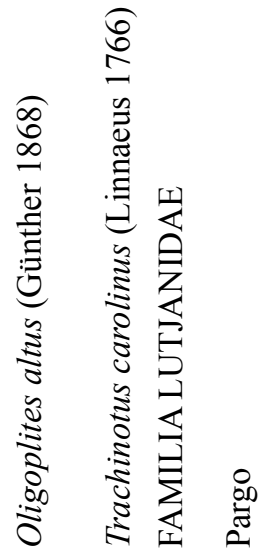

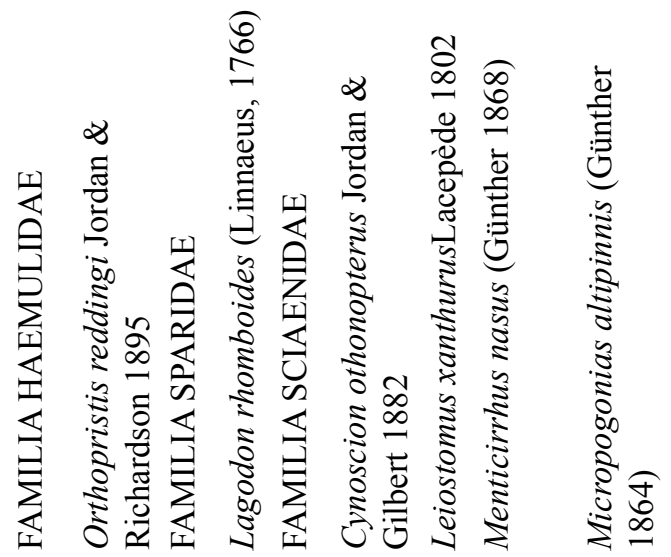

Rev. Mar. Cost. Vol. 12 (1): 99-115, enero-junio 2020.

ISSN: $1659-455 \mathrm{X} \cdot \mathrm{e}-\mathrm{ISSN}$ : $1659-407 \mathrm{X}$

DOI: http://dx.doi.org/10.15359/revmar.12-1.5 

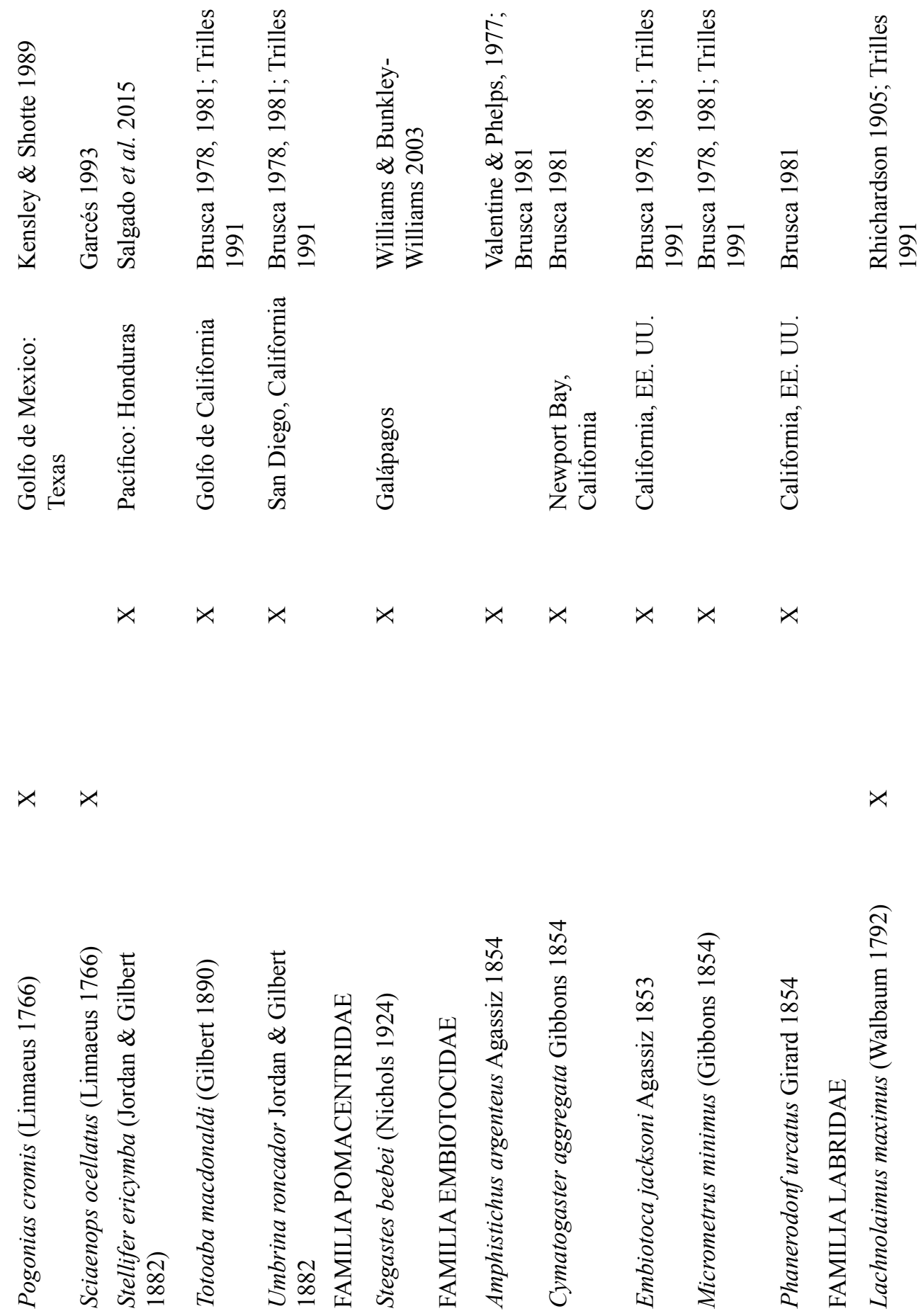


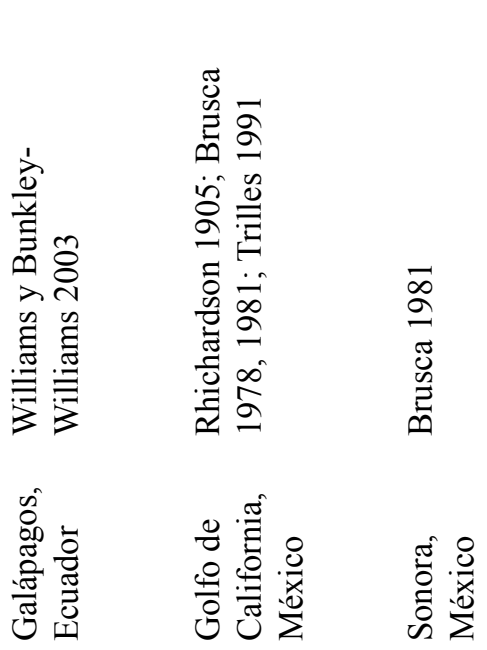

$x$

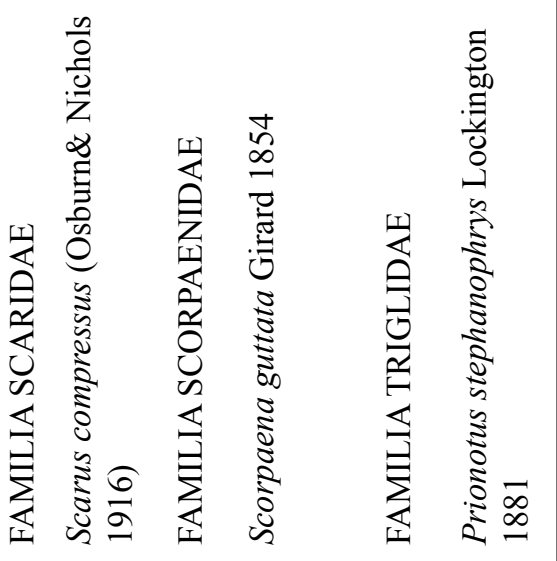

\section{DISCUSIÓN}

En el Atlántico occidental existen reportes de isópodos que parasitan a elasmobranquios (Bird, 1978; Shipley et al. 2017). Aunque la mayoría son ectoparásitos, se ha reportado al isópodo carroñero Cirolana sp., en la cavidad corporal del tiburón cabeza de pala Sphyrna tiburo, en la costa de Miramar, La Habana, Cuba (Ortiz et al. 2018). También, se han detectado cimotoideos que parasitan elasmobranquios en aguas continentales; en la cuenca del Orinoco venezolano se registró a Braga cigarra y Exocorallana delaneyi en rayas del género Potamotrygon (Lasso et al. 2018). Mientras, en la región suroeste del golfo de México, cercana al área del presente registro, se describió Anilocra elviae, un ectoparásito del tiburón Isurus oxyrinchus (Winfield et al. 2002).

A pesar de los registros señalados anteriormente, estos suelen ser aislados, por lo que resulta raro el parasitismo de cimotoideos con los elasmobranquios. Es posible que dicha asociación se deba a la unión temporal de crías o de ejemplares que han escapado de su hospedero cuando son apresados dentro de las capturas comerciales (Brusca, 1981), como pudo suceder con el registro de $N$. californica en ejemplares de Pseudobatos glaucostigma, obtenidos de la pesca de arrastre de camarón en el sureste del golfo de California (Carrillo-Colín 
et al. 2016). Además, algunas especies como $N$. acuminata tienen la capacidad de cambiar de hospedero (Williams et al. 1982).

Las escamas placoideas presentes en la mayoría de los elasmobranquios les brindan protección en contra de agentes externos, mediante un endurecimiento de la pared corporal, lo que impide la penetración de las piezas bucales de algunas especies de Cymthoidae (Brusca, 1981). En cambio, son más susceptibles al parasitismo por parte de copépodos y monogéneos, los cuales se adhieren en una región corporal determinada (Caira et al. 2012), lo que ocasiona incluso lesiones cutáneas crónicas que afectan la salud de los tiburones (Bullard et al. 2000).

Por otro lado, en las etapas juveniles de elasmobranquios, inclusive las cápsulas ovígeras y aquellas especies que carecen de dentículos, son susceptibles a ser parasitados por isópodos cimotoideos, los cuales suelen alimentarse de sangre y fluidos internos. En el caso de N. acuminata, se ha comprobado de manera experimental que no muestra especificidad con un hospedero y que suele atacarlos desde el fondo o a media agua (Segal, 1987). Al contrastar la diversidad de hospederos, existe un predominio de peces Actinopterigios, 26 especies asociadas con $N$. acuminata y 41 para $N$. californica. La mayoría de este grupo de peces pertenecen a familias de hábitos demersales, como Sciaenidae y
Serranidae. De manera general, estos isópodos aparentan tener preferencia por los peces óseos (Trilles \& Öktener, 2004; Williams et al. 2010).

\section{AGRADECIMIENTOS}

Al proyecto PAPIIT-UNAM IA207820 por el financiamiento proporcionado, así como al SIN-CONACYT. Extendemos nuestro agradecimiento a la corporativa pesquera "Roca" y a todo su personal, en especial a Constantino Pio Campos "Titán" quienes facilitaron el soporte técnico durante el trabajo de campo, así como las fotografías del arte de pesca. A los revisores anónimos que ayudaron a mejorar la presente contribución.

\section{REFERENCIAS}

Álvarez-León, R. (1981). El isópodo Nerocila californica, simbionte de Cytarichthys gilberti (Bothidae) en el sistema lagunar de Huizache-Caimanero, Sinaloa, México. Rev. Biol. Trop., 29(1), 39-44.

Benz, G. W. \& Bullard, S. A. (2004). Metazoan parasites and associates of chondrichthyans with emphasis on taxa harmful to captive hosts. En M. Smith \& D. Warmolts, D. Thoney (Eds.), Elasmobranch husbandry manual: captive care of shark, rays and their relatives (pp. 325-416). EE. UU.: Ohio Biological surveys.

Bird, P. M. (1978). The occurrence of Cirolana biorealis (Isopoda) in the hearts of sharks from Atlantic Coastal waters of Florida. Fish Bull., 79(2), 376-383. 
Boyko, C. B., Bruce, N. L., Hadfield, K. A., Merrin, K. L., Ota, Y., Poore, G. C. B. \& Wilson, G. D. F. (2020). World Marine, Freshwater and Terrestrial Isopod Crustaceans database. http://www.marinespecies.org/isopoda

Brusca, R. C. (1978). Studies on the cymothoid fish symbionts of the eastern Pacific (Isopoda: Cymothoidae) I. Biology of Nerocila californica. Crustaceana, 34(2), 141-154.

Brusca, R. C. (1981). A monograph on the Isopoda Cymothoidae (Crustacea) of the eastern Pacific. Zool. J. Linn. Soc., 73, 117- 199.

Brusca, R. C. \& Iverson, E. W. (1985). A guide to the Marine Isopod Crustacea of Pacific Costa Rica. Rev. Biol. Trop., 33(1), 1-77.

Bullard, S. A., Frasca, S. \& Benz G. W. (2000). Skin lesions caused by Dermophthirius penneri (Monogenea: Microbothriidae) on wild-caught blacktip Sharks (Carcharhinus limbatus). J. Parasitol., 86(3), 618-622.

Bunkley-Williams, L. \& Williams, E. H. Jr. (1999). Nerocila benrosei n. sp. (Isopoda: Cymothoidae), an external parasite of hogfishes from the norther Bahamas.J. Parasitol., 85(6), 1036-1040.

Caira, J. M. \& Healy, C. J. (2004). Elasmobranchs as hots of metazoan parasites in biology of sharks and their relatives. In: J. C. Carrier, J. A. Musick \& M. R. Heithaus (Eds), Biology of sharks and their relatives (523-551). $1^{\text {st }}$ Edition. EE. UU. CRC Press.

Caira, J. M., Healy, C. J. \& Jensen, K. (2012). An updated look at elasmobranchs as Hosts of metazoan parasites. In J. C. Carrier, J. A. Musick \& M. R. Heithaus (Eds.), Biology of sharks and their relatives (546-557). $2^{\text {nd }}$ Edition. EE. UU.: CRC Press.
Carrillo-Colín, D. L., Lara-Mendoza, R. E. \& Márquez-Farías, J. (2016). Nerocila acuminata (Crustacea: Isopoda: Cymothoidae), ectoparásito de la guitarra pinta Pseudobatos glaucostigma (Elasmobranchii) del sureste del Golfo de California, México. Cien. Pesq., 24, 139-143.

Fricke, R., Eschmeyer, W. N. \& van der Laan, R. (2020). Echmeyer's catalog of fishes: genera, species, references. Recuperado en noviembre 8, 2020, disponible en http://researcharchive. calacademy.org/research/ichthyology/ catalog/fishcatmain.asp

Garcés, H. A. (1993). Primer informe en Panamá de Nerocila californica Schioedte y Meinert 1881 (Isopoda: Cymothoidea) en Sciaenops ocellatus (L) (Pisces: Sciaenidae). Rev. Med. Panama, 18(1), 70-73.

Hutton, R. F. (1964). A second list of parasites from marine and costal animals of Florida. Trans. Am. Microsc. Soc., 83(4), 439-447.

Kensley, B. \& Schotte, M. (1989). Marine isopod crustaceans of the Caribbean. EE. UU.: Smithsonian Institution Press.

Lasso, C. A., Campos, M. R., Morales-Betancourt, M. A. \& Castro, D. (2018). Ectoparásitos (Argulidae, Cymothoidae, Corallanidae) en rayas de agua dulce (Potamotrygonidae) de la Orinoquia colombiana. Biota Colomb., 19 (Supl. 1), 84-94.

McEachran, D. J. \& Fechhelm, D. J. (1998). Fishes of the Gulf of Mexico. Volumen 1: Myxiniformes to Gasterosteiformes. EE. UU.: University of Texas Press.

Moreira, P. S. \& Sadowsky, V. (1978). An annotated bibliography of parasitic Isopoda (Crustacea) of Chondrichthyes. Bol. Inst. Oceanogr. Sao Paulo, 27(2), 95-152. 
Ortiz, M., Lalana, R. \& Torres, O. (2018). Primer registro de un isópodo carroñero del género Cirolana Leach, 1818 (Crustacea, Isopoda) colectado de la cavidad corporal de Sphyrna tiburo (Elasmobranchii, Sphyrnidae), en Cuba. Rev. Inv.Mar., 38(1), 45-50.

Pearse, A. S. (1952). Parasitic Crustacea from the Texas Coast. Publs. Inst. Mar. Sci. Univ. Tex., 2(2), 6-42.

Salgado, A. I., Mérida, J. E. \& Cruz, G. A. (2015). Los isópodos Cymothoa exigua y Nerocila acuminata (Isopoda: Cymothoidae), ectoparásitos de Parapsettus panamensis (Ephippidae), Chloroscombrus orqueta (Carangidae) y Stellifer ericymba (Sciaenidae) del Pacífico de Honduras. Cuad. Inv. UNED, 7(2), 301-304.

Segal, E. (1987). Behavior of juvenile Nerocila acuminata (Isopoda, Cymothoidae) during attack, attachment and feeding on fish prey. Bull. Mar. Sci., 4l(2), 351-360.

Shipley, O., Talwar, B., Grubbs, D. \& Brooks, E. (2017). Isopods present on deep-water sharks Squalus cubensis and Heptranchias perlo from The Bahamas. Mar. Biodivers., 47(3), 789-790.

Smit, N. J., Bruce, N. L. \& Hadfield, K. A. (2014). Global diversity of fish parasitic isopod of the family Cymothoidae. Int. J. Parasitol. Parasites Wildl., 3(2), 188-197.

Trilles, J. P. (1991). Les Cymothoidae (Crustacea, Isopoda) du monde. Prodrome pour une faune. Stud. Mar., 21-22(1-2), 5-288.

Trilles, J. P. \& Öktener, A. (2004). Livoneca sinuate (Crustacea; Isopoda,Cymothoidae) on Loligo vulgari from Turkey, an unusual cymothoid association. Dis. Aquat. Org., 61(3), 235-240.

Trilles, J. P., Rameshkumar, G. \& Rachichandran, S. (2013). Nerocila species
(Crustacea, Isopoda, Cymothoidae) from Indian marine fishes. Parasitol. Res., 112(3), 1273-1286.

Valentine, D. W. \& Phelps, R. (1977). Parasitism of barred surperch, Amphistichus argenteus, by Nerocila californica: a fit to the poisson. Cal. Fish Game, 63(2), 129-132.

van der Laan, R., Fricke, R. \& Eschmeyer, W. N. (2019). Eschmeyer' catalog of fishes: clasification. http:// www.calacademy.org/scientists/ catalog-of-fishes-classification/

Williams, E. H. \& Bunkley-Williams, L. (2003). New records of fish-parasitic isopods (Cymothoidae) in the Eastern Pacific (Galápagos and Costa Rica). Noticias de Galápagos, 62, 21-23.

Williams, E. H., Bunkley-Williams, L. \& Ebert, D. E. (2010). An accidental attachment of Eltusa raynaudii (Isopoda, Cymohoidae) in Etmopterus sp. (Squaliformes Etmopteridae). Acta Parasitol., 55(1), 99-101.

Williams E. H., Jr., Bunkley-Williams L., Waldner, R. E. \& Kimmel, J. J. (1982). Predisposition of a pomacentrid fish, Chromis multilineatus (Guichenot) to parasitism by a cymothoid isopod, Anilocra chromis Williams and Williams. J. Parasitol., 68(5), 942-945.

Winfield, I., Alvarez, F. \& Ortiz M. (2002). A new species of Anilocra (Crustacea: Isopoda: Cymothoidae), ectoparasitc on the mako sharks Isurus oxyrinchus. Proc. Biol. Soc. Wash., 115(1), 148-152. 
